\title{
Quantitative analyses of essential fatty acids in cereals and green vegetables by isotope dilution-gas chromatography/mass spectrometry
}

\author{
Sunyoung Lee ${ }^{1}$, Dong Kyu Lim', Song-Yee Baek', Dongwon Seo², Jong-Su Park³, Byung-Man Kwak , \\ Jongeun Won ${ }^{3}$, Joonhee Lee ${ }^{1 * \dagger}$ and Byungjoo $\mathrm{Kim}^{1+}$
}

\begin{abstract}
An analytical method for the accurate determination of essential fatty acids in cereals and green vegetables is established based on isotope dilution-gas chromatography/mass spectrometry (ID-GC/MS). In this approach, ${ }^{13} \mathrm{C}$ labeled fatty acids were added as internal standards to the samples prior to the sample preparation. Repeatability and reproducibility for the analyses of the essential fatty acids extracted from homogenized cereals and green vegetables were examined to validate the ID-GC/MS method. The repeatability and reproducibility were within $2 \%$ relative standard deviation. The developed method provides higher-order measurement results with the relative expanded uncertainties of $1-2 \%$ and $2-4 \%$ for cereals and green vegetables, respectively. Based on the fatty acid measurements, the developed ID-GC/MS method can be used as a candidate reference method for the quantitation of essential fatty acids in cereals and green vegetables. The developed method was also applied to analyze several commercially available products including cereal and vegetable powders.
\end{abstract}

Keywords: Essential fatty acid, Isotope-labeled fatty acid, Isotope dilution-gas chromatography/mass spectrometry, Cereal, Green vegetable

\section{Introduction}

Essential fatty acids are present in various foods as metabolites and intermediates of diverse biological processes as well as the main source of energy in human beings.(Harwood, 1988; Innis, 2008; Holman, 1998; Arbuckle et al., 1994; Neuringer et al., 1984) For humans, dietary intake is the only way to obtain essential fatty acids such as $\alpha$-linolenic acid ( $\mathrm{n}-3$ fatty acid) and linoleic acid (n-6 fatty acid).(Innis, 1991) The low intake of essential fatty acids has a negative effect on human health.(Innis, 2008; Uauy \& Dangour, 2006) It is reported that $\mathrm{n}-3$ fatty acid deficiency leads to decreased

\footnotetext{
* Correspondence: joonhee@kriss.re.kr

${ }^{\dagger}$ Joonhee Lee and Byungjoo Kim contributed equally to this work. 'Division of Chemical and Biological Metrology, Korea Research Institute of Standards and Science (KRISS), Daejeon 34113, Republic of Korea Full list of author information is available at the end of the article
}

visual and neural development in infants and children, and the deficiency of n- 6 fatty acid causes poor growth and reproductive failure.(Innis, 2008; Connor, 1999) Therefore, reliable fatty acid measurements of foods are required for adequate intake of fatty acids. Additionally, the quantitative analysis of fatty acids is needed to characterize the nutritional quality of food.

Cereals and vegetables are our staple foods that provide essential dietary nutrients including fatty acids. Analysis of fatty acids in these foods has been investigated using various analytical techniques,(Yoshida et al., 2011; Yasumatsu \& Moritaka, 1964; Komaitis \& Melissari-Panagiotou, 1990; Lee et al., 2000) of which gas chromatography (GC) approaches have been the most widely utilized methods. Although GC analysis requires esterification and methylation processes to form fatty acid methyl esters (FAMEs) to increase the 
volatility and decrease the polarity of compounds for GC measurements, GC coupled with mass spectrometry (MS) provides the most definitive information for the identification and quantification of fatty acids.(Lee et al., 2019a; Takemoto et al., 2003; Cruz-Hernandez et al., 2013) In many cases, a single FAME as an internal standard is added to the sample for the quantitative analysis of fatty acids,(Takemoto et al., 2003; CruzHernandez et al., 2013) which presents limitations in an appropriate correction of recovery during the derivatization process as well as instrument sensitivity owing to the different performances of the reactions and measurements of various fatty acids. The Comité Consultatif pour la Quantité de Matière (CCQM) identified that an isotope dilution mass spectrometry (IDMS) approach has a potential to be a primary method in chemistry.(Quinn, 1997; Richter, 1997) Therefore, in this study, an isotope dilution-gas chromatography/mass spectrometry (ID-GC/MS) method is established as a higher-order reference method, in which an isotope-labeled fatty acid is used to correct the recovery and detection sensitivity of the target fatty acid during the sample preparation steps and instrumental measurements. The developed ID-GC/ MS method affords metrologically traceable measurement results and ensures accurate determination of fatty acid content in foods.

The aim of this study is to develop an accurate analytical method as a higher-order reference method using IDMS for fatty acid analysis in the food. The developed ID-GC/MS method as a primary method provides measurement results with traceability to the International System (SI) of units. Our research laboratory at Korea Research Institute of Standards and Science as the National Metrology Institute of Korea has been developing food-matrix certified reference materials (CRMs) along with a higher-order reference method under a project of nutritional metrology program.(Lee et al., 2017; Lee et al., 2016; Lee et al., 2019b; Shin et al., 2013) The food-matrix CRMs provide certified values for the target nutrients and can be used to verify the nutrient measurements. Under this program, the IDMS methods have been developed as candidate reference methods for the quantitative analysis of individual nutrients in various foods. Recently, accurate amounts of several fatty acids in infant formula were determined using the developed IDMS approach by our research group.(Lee et al., 2019a) There are a few food CRMs to provide certified values for nutrients including fatty acids.(Lee et al., 2019b; Wise \& Phillips, 2019; Sharpless et al., 2019) However, to the best of our knowledge, no CRM for the essential fatty acid analysis in cereal or vegetable matrix is currently available. As cereals and vegetables are highly consumed foods in the diet, which are the main source of nutrition including fatty acids, the IDMS method is expanded to analyze the essential fatty acid content in raw food matrices in the present study. The repeatability and reproducibility of the developed IDMS method for the analysis of essential fatty acids in cereals and green vegetables are evaluated. As a result, the repeatability and reproducibility were within $2 \%$ relative standard deviation for all samples. The developed ID-GC/MS method provides higher-order metrological measurement values, which can be a candidate reference method.

\section{Experimental}

\section{Materials and chemicals}

Linoleic acid and $\alpha$-linolenic acid were purchased from MilliporeSigma Corporate (St. Louis, MO, USA). These fatty acids were used as primary reference materials for sample analysis. The purity assays of the fatty acids were performed according to the protocols based on a massbalance approach, maintained in the laboratory at the National Metrology Institute of Korea.(Kim et al., 2013; Lee \& Kim, 2014) The purity determination can be applied to the primary reference materials for linking traceability of their certified values of target organic substances in matrix CRMs to the SI units. The purities of linoleic acid and $\alpha$-linolenic acid were determined to be $96.2 \pm 1.1 \%$ and $99.1 \pm 0.2 \%$, respectively. ${ }^{13} \mathrm{C}_{18}$-linoleic acid and ${ }^{13} \mathrm{C}_{18}$ - $\alpha$-linolenic acid were obtained from Medical Isotopes Inc. (Pelham, NH, USA). Anhydrous sodium sulfate, butylated hydroxytoluene (BHT), hydrochloric acid, sodium chloride, sodium hydroxide, $14 \%$ boron trifluoride in methanol solution, heptane, and petroleum ether were obtained from MilliporeSigma Corporate. Chloroform, ethanol, methanol, and n-hexane were purchased from Honeywell Burdick \& Jackson (Muskegon, MI, USA). Cereal and green vegetable samples were purchased from the local markets.

\section{Standard solutions}

Standard solutions and isotope ratio standard solutions were prepared by the procedure maintained in our laboratory as described in previous studies.(Lee et al., 2019a; Kim et al., 2010) Four standard solutions containing approximately $500 \mathrm{mg} / \mathrm{kg}$ of the target fatty acid(s) were gravimetrically prepared by dissolving each fatty acid in ethanol including $0.1 \%$ BHT as an antioxidant. An isotope-labeled standard solution containing ${ }^{13} \mathrm{C}_{18^{-}}$ linoleic acid (and ${ }^{13} \mathrm{C}_{18}$ - $\alpha$-linolenic acid in case of vegetable samples) was also prepared in the same manner. For each standard solution, two isotope ratio standard solutions were prepared by gravimetrically mixing it with the isotope-labeled solution (1:1 isotope ratio). A total of eight isotope ratio standard solutions was analyzed by GC/MS to evaluate the repeatability of the preparing standard solutions and isotope ratio standard solutions. 
One isotope ratio standard solution was selected as a calibration standard for sample analysis.

\section{Lipid extraction, saponification, and methylation}

Approximately $0.3 \mathrm{~g}$ of each sample was added into a $50-\mathrm{mL}$ glass tube and spiked with the isotope-labeled standard solution to obtain a 1:1 ratio of the isotopelabeled fatty acid to the target essential fatty acid. To extract the bound lipid from the grain samples, acid hydrolysis using the procedure described by Taylor et al.(Taylor \& Nelson, 1920) was performed by adding $5 \mathrm{~mL}$ of $1 \mathrm{~N} \mathrm{HCl}$ solution to the glass tube and heating at $100^{\circ} \mathrm{C}$ in a hot water bath for $30 \mathrm{~min}$. After cooling the sample, $3 \mathrm{~mL}$ of $3 \mathrm{~N} \mathrm{NaOH}$ solution was added. Thereafter, $10 \mathrm{~mL}$ of chloroform-methanol $(2: 1, \mathrm{v} / \mathrm{v})$ mixture was added in the case of cereal samples according to the Folch procedure, and the sample tube was heated at $100{ }^{\circ} \mathrm{C}$ for $30 \mathrm{~min}$ to extract the free lipids.(Folch et al., 1957) For green vegetable samples, $10 \mathrm{~mL}$ of $n$-hexane was added to the sample tube and the sample solution was heated at $60^{\circ} \mathrm{C}$ for $1 \mathrm{~h}$.

After lipid extraction, saponification and methylation reactions were performed to obtain FAMEs for GC/MS analysis. The content in the sample tube was mixed with $5 \mathrm{~mL}$ of $0.5 \mathrm{~N} \mathrm{NaOH}$ in methanol and heated at $100{ }^{\circ} \mathrm{C}$ for $10 \mathrm{~min}$ to obtain free fatty acids (FFAs). Then, the FFAs were derivatized using $5 \mathrm{~mL}$ of $14 \%$ boron trifluoride in methanol at $100^{\circ} \mathrm{C}$ for $5 \mathrm{~min}$. After adding $10 \mathrm{~mL}$ of the saturated sodium chloride solution, $5 \mathrm{~mL}$ of heptane and $10 \mathrm{~mL}$ of petroleum ether were used to extract the FAMEs. The extract (upper organic layer) was transferred into another tube, and residual water was removed by adding sodium sulfate. The final extract was dried at $50{ }^{\circ} \mathrm{C}$ under nitrogen gas and diluted with $n$ hexane to obtain a concentration of ppm level suitable for GC/MS analysis.

\section{ID-GC/MS instrument conditions and calculations}

A triple quadrupole mass spectrometer with a GC system (TSQ8000 and TRACE1310, Thermo Fisher Scientific, Waltham, MA, USA) was utilized for ID-GC/MS analysis. The chromatographic separation of FAMEs was performed using a HP-88 capillary column (length $60 \mathrm{~m}$, ID $0.25 \mathrm{~mm}$, film thickness $0.2 \mu \mathrm{m}$, Agilent Technologies, Inc., Santa Clara, CA, USA). Helium at a constant flow rate of $1.0 \mathrm{~mL} / \mathrm{min}$ was used as the carrier gas. The injector temperature was set to $250{ }^{\circ} \mathrm{C}$ and the sample was introduced into the GC in splitless mode with a splitless time of $0.5 \mathrm{~min}$. The oven temperature was programmed from $80^{\circ} \mathrm{C}$ to $180^{\circ} \mathrm{C}$ at a rate of $40^{\circ} \mathrm{C} / \mathrm{min}$ and maintained for $14 \mathrm{~min}$. The final oven temperature was increased to $230^{\circ} \mathrm{C}$ at a rate of $5{ }^{\circ} \mathrm{C} / \mathrm{min}$ and held for $5 \mathrm{~min}$. The temperatures of the mass transfer line and ion source were $230^{\circ} \mathrm{C}$ and $200{ }^{\circ} \mathrm{C}$, respectively.
FAMEs were ionized in the electron impact ionization (EI) mode employing $70 \mathrm{eV}$ of electron energy. The quantitative analysis of FAMEs was performed using the selected ion monitoring (SIM) mode. The molecular ions of methylated ester forms of essential fatty acids including methyl linoleate and $\alpha$-methyl linolenate with their isotopic analogues, ${ }^{13} \mathrm{C}_{18}$-methyl linoleate and ${ }^{13} \mathrm{C}_{18}-\alpha$ methyl linolenate, were detected at $\mathrm{m} / \mathrm{z}=294.2,292.2$, 312.2 , and 310.2, respectively.

A detailed description of the calculation and uncertainty evaluation applied for the quantitative analysis of fatty acids through ID-GC/MS measurements has been reported in previous studies.(Kim et al., 2010; Choi et al., 2003) The mass fractions of the individual fatty acids $\left(C_{\text {sample }}\right)$ in cereals and green vegetables $(\mathrm{mg} / \mathrm{kg})$ were calculated using the following equation.

$$
C_{\text {sample }}=\frac{M_{\text {is }- \text { sol,spiked }} \cdot A R_{\text {sample }} \cdot M_{s-\text { sol,std.mix. }} \cdot C_{s-\text { sol }}}{M_{\text {sample }} \cdot A R_{\text {std.mix. }} \cdot M_{\text {is }- \text { sol,std.mix. }}}
$$

Here, $M_{\text {is-sol, spiked }}$ is the mass of the isotope-labeled fatty acid solution added to the sample, $A R_{\text {sample }}$ indicates the ratio of the FAME and isotope-labeled FAME peak areas in the GC/MS chromatogram of the test sample, $M_{s \text {-sol,std.mix. }}$ and $M_{\text {is-sol,std.mix. }}$ are the masses of the fatty acid standard solution and isotope-labeled fatty acid solution, respectively, added to the isotope ratio standard solution, $C_{s-\text { sol }}$ corresponds to the concentration of the fatty acid in the fatty acid standard solution, $M_{\text {sample }}$ is the mass of the sample subjected to analysis, $A R_{\text {std.mix. }}$ corresponds to the ratio of the FAME and isotope-labeled FAME peak areas observed in the GC/ MS chromatogram of the isotope ratio standard solution.

\section{Results and discussion \\ Fatty acid analyses by ID-GC/MS}

Homogenized cereal and green vegetable samples, which were developed as candidate CRMs in our laboratory, were tested to determine the overall fatty acid contents in the scan mode of GC/MS. We have used the HP-88 column which shows a good separation for the FAMEs in our previous studies.(Lee et al., 2019a; An et al., 2013) The total ion chromatograms (TICs) using the HP-88 column were obtained under optimized extraction and GC conditions described in the experimental section. Figure 1 shows the TICs of the FAMEs extracted from rice and wheat flours, as well as spinach and Kimchi cabbage powders. The peaks in the TICs were identified based on the mass spectrum library and GC/MS analysis of the fatty acid standard solutions. As shown in Fig. 1, the cereal samples predominantly contain palmitic acid, which is a saturated fatty acid. Linoleic acid is present as a dominant essential fatty acid component in both cereal 


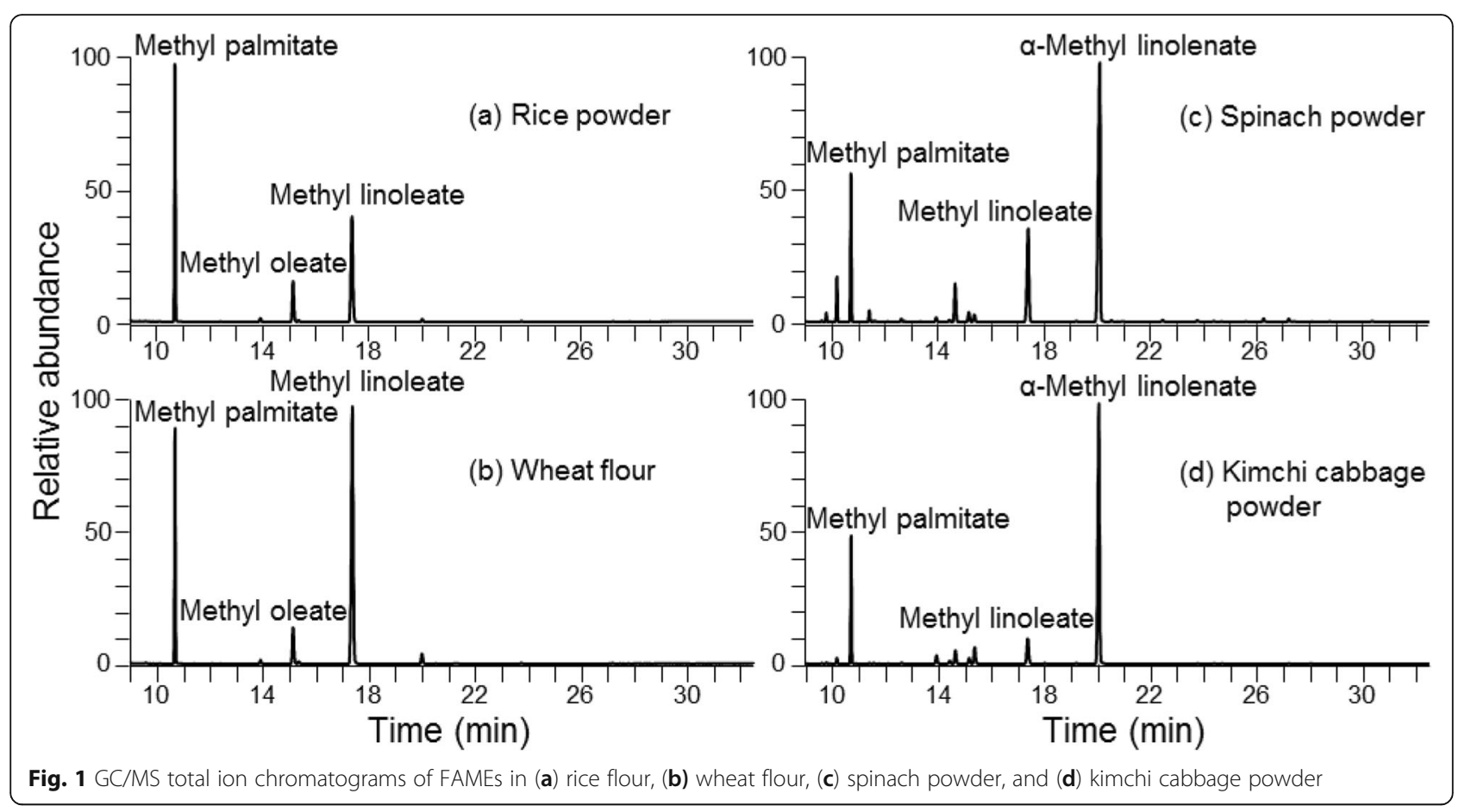

lipids. The results agree with those reported in previous studies.(Yoshida et al., 2011; Yasumatsu \& Moritaka, 1964) Wheat flour contains a higher content of linoleic acid than that present in the rice flour. Each cereal sample also contains a low content of $\alpha$-linolenic acid. For the green vegetable samples, spinach and Kimchi cabbage (which is the main ingredient for Kimchi) were examined. Two essential fatty acids, linoleic acid and $\alpha-$ linolenic acid, are present as major components in the vegetables. Lee et al. also reported that a high content of $\alpha$-linolenic acid presents in the spinach lipids.(Lee et al., 2000) The vegetable oils have a higher content of unsaturated fatty acids than those contained in the cereal oils. In particular, $\alpha$-linolenic acid is the most abundant fatty acid in both vegetable samples.

In this study, the essential fatty acids with high contents, i.e., linoleic acid in the cereal samples and linoleic acid and $\alpha$-linolenic acid in the vegetable samples, were quantitatively analyzed using ID-GC/MS. Figure 2 shows the SIM chromatograms of the target FAMEs and their isotope-labeled analogues, which were derivatized from rice flour, wheat flour, and spinach powder extracts. A previous study by our research group demonstrated that molecular ion intensities of the target FAMEs with relatively short carbon chains $(<\mathrm{C} 20)$ and three or less $\mathrm{C}=\mathrm{C}$ double bonds were sufficient for monitoring in the EI mode.(Lee et al., 2019a) Methyl linoleate and ${ }^{13} \mathrm{C}_{18}$-methyl linoleate are both observed at $17.7 \mathrm{~min}$, and methyl linolenate and ${ }^{13} \mathrm{C}_{18}$ - $\alpha$-methyl linolenate are both detected at $20.3 \mathrm{~min}$ by monitoring the molecular ions of
FAMEs and their isotope-labeled forms. No noticeable interference from the sample matrix is observed. As mentioned above, the fatty acid content was calculated using the ratio of the observed FAME and its isotopelabeled analogue peak areas.

\section{Method validation}

As neither well-characterized reference material nor fortified sample for comparison is available, the validation of the developed ID-GC/MS method as a highorder reference method was tested by evaluating the repeatability, reproducibility, and uncertainty of target fatty acid analysis in the homogenized cereal and green vegetable samples. The repeatability of the IDGC/MS method was assessed by analyzing three subsamples of each candidate reference material within a day. To determine the reproducibility of the developed approach, the same repeatability test for the individual sample was performed on three different days. The measurement results for the target fatty acids in the cereal and green vegetable samples with expanded uncertainties are listed in Tables 1 and 2, respectively. The relative standard deviations (RSD) of the repeatability tests using the ID-GC/MS method are in the $0.23-1.61 \%$ range for the cereal samples and $0.39-1.89 \%$ range for the vegetable samples. Therefore, the developed method demonstrates good repeatability for fatty acid analysis. The RSDs of the mean values of linoleic acid content obtained at three different time periods are 1.48 and $0.95 \%$ for rice and 


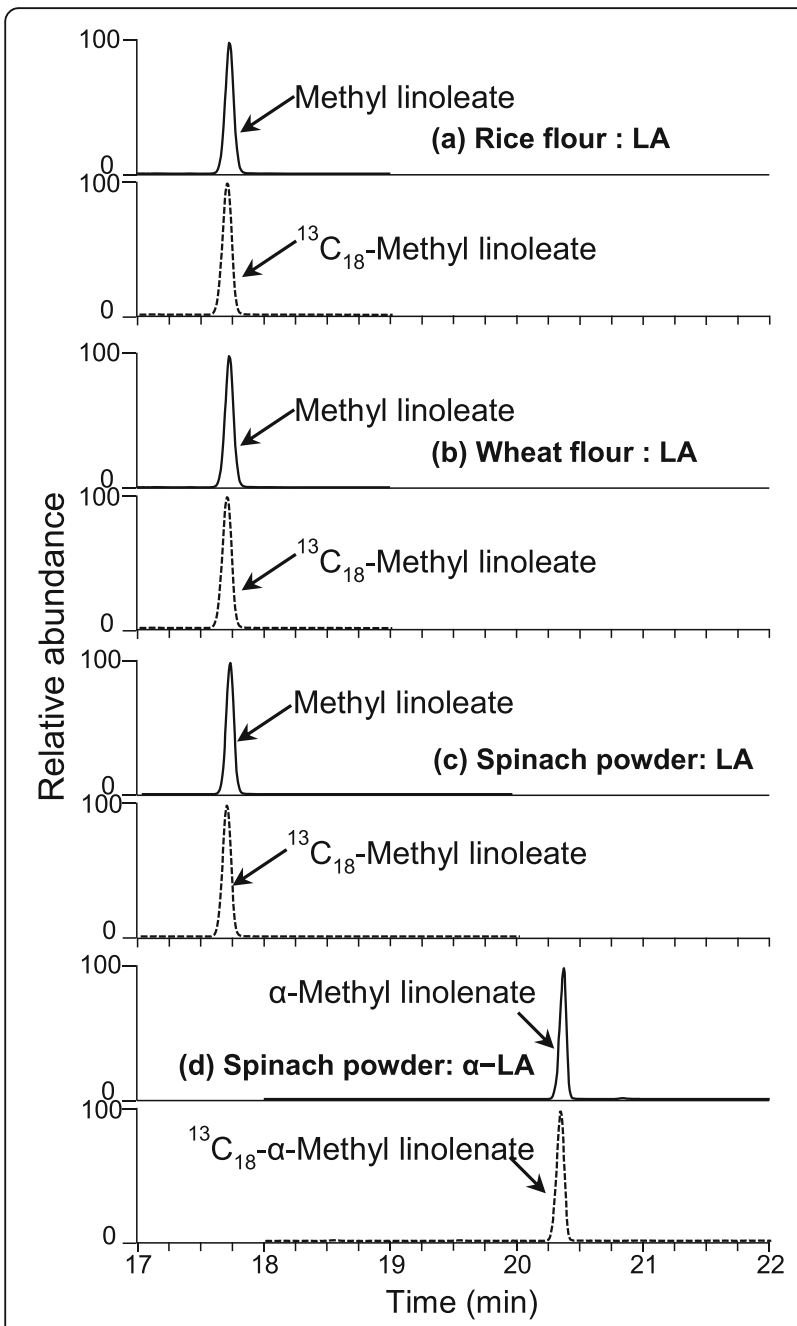

Fig. 2 SIM chromatograms of methyl linoleate and ${ }^{13} \mathrm{C}_{18}$ methyl linoleate extracted from (a) rice flour, (b) wheat flour, and (c) spinach powder and (d) SIM chromatograms of a-methyl linolenate and ${ }^{13} \mathrm{C}_{18}$-a-methyl linolenate extracted from spinach powder

wheat flours, respectively. The RSDs of the measurement values for spinach and Kimchi cabbage powders show similar results $(\leq 1.6 \%)$, which indicate good reproducibility of the developed method. The relative expanded uncertainties with a confidence level of $95 \%$ for the cereal and vegetable samples are between 1 and $4 \%$, as shown in Tables 1 and 2 . The small range of the measurement uncertainties indicates that the developed ID-GC/MS approach can be used as a higher-order reference method for the precise and accurate analysis of the target fatty acids.

As mentioned above, the well-characterized reference material including any fatty acid value with cereal or vegetable matrix is not available for comparison with measured values by the developed method. In this study, an interlaboratory comparison among collaborating laboratories, including Namyang
Table 1 Results of ID-GC/MS measurements for homogenized samples of rice and wheat flours at three different time periods

\begin{tabular}{|c|c|c|c|}
\hline \multirow[t]{3}{*}{ Periods } & \multirow{3}{*}{$\begin{array}{l}\text { Subsample } \\
\text { No. }\end{array}$} & \multicolumn{2}{|c|}{ Results by ID-GC/MS (mg/kg) ${ }^{a}$} \\
\hline & & Rice flour & Wheat flour \\
\hline & & Linoleic acid & Linoleic acid \\
\hline \multirow[t]{5}{*}{1} & 1 & $3599 \pm 64$ & $8341 \pm 130$ \\
\hline & 2 & $3717 \pm 71$ & $8254 \pm 129$ \\
\hline & 3 & $3660 \pm 54$ & $8335 \pm 112$ \\
\hline & Mean & 3659 & 8310 \\
\hline & $S D^{b}$ & $59(1.61 \%)$ & $49(0.58 \%)$ \\
\hline \multirow[t]{5}{*}{2} & 1 & $3538 \pm 43$ & $8400 \pm 88$ \\
\hline & 2 & $3554 \pm 59$ & $8494 \pm 96$ \\
\hline & 3 & $3573 \pm 58$ & $8437 \pm 102$ \\
\hline & Mean & 3555 & 8437 \\
\hline & $S D^{b}$ & $18(0.49 \%)$ & $50(0.59 \%)$ \\
\hline \multirow[t]{5}{*}{3} & 1 & $3623 \pm 67$ & $8474 \pm 120$ \\
\hline & 2 & $3616 \pm 43$ & $8461 \pm 170$ \\
\hline & 3 & $3650 \pm 61$ & $8436 \pm 115$ \\
\hline & Mean & 3630 & 8457 \\
\hline & $S D^{b}$ & $18(0.49 \%)$ & $19(0.23 \%)$ \\
\hline \multicolumn{2}{|c|}{ Overall mean } & 3614 & 8401 \\
\hline \multicolumn{2}{|c|}{ SD among periods ${ }^{c}$} & $53(1.48 \%)$ & $80(0.95 \%)$ \\
\hline
\end{tabular}

The number following " \pm " is the expanded uncertainty with a $95 \%$ confidence level for the measurement value.

${ }^{\mathrm{b}}$ The standard deviation (SD) was determined among three values obtained within a day.

${ }^{\mathrm{C}}$ The standard deviation (SD) was determined among three mean values obtained at different time points.

Dairy Products Company and Korea Food Research Institute (KFRI), was carried out to evaluate conformity and commutability of the ID-GC/MS method for the analysis of homogenized rice sample. The collaborating laboratories used a gas chromatography-flame ionization detection (GC-FID) approach based on Method 2.1.5.4 in the Korean food code. The GC-FID method used another FAME as an internal standard, which cannot eliminate possible bias resulted from inappropriate recovery correction during sample preparation steps and improper sensitivity calibration during instrumental measurements. The developed ID-GC/ MS method as a primary method provides measurement results with traceability to the SI of units, which ensures the recovery correction and instrument sensitivity. The linoleic acid content in multiple subsamples $(n=10)$ of rice using the developed method was $3621 \pm 17 \mathrm{mg} / \mathrm{kg}$ (RSD = 0.5\%). The values obtained from Namyang and KFRI laboratories were $3478 \pm 191 \mathrm{mg} / \mathrm{kg}(\mathrm{RSD}=5.5 \%)$ and $3603 \pm 64 \mathrm{mg} /$ $\mathrm{kg}(\mathrm{RSD}=1.8 \%)$, respectively. The measurement results by the developed method are comparable to those obtained by the collaborating laboratories. This 
Table 2 Results of ID-GC/MS measurements for homogenized samples of spinach and kimchi cabbage powders at three different time periods

\begin{tabular}{|c|c|c|c|c|c|}
\hline \multirow[t]{3}{*}{ Periods } & \multirow{3}{*}{$\begin{array}{l}\text { Subsample } \\
\text { No. }\end{array}$} & \multicolumn{4}{|c|}{ Results by ID-GC/MS (mg/kg) ${ }^{a}$} \\
\hline & & \multicolumn{2}{|c|}{ Spinach powder } & \multicolumn{2}{|c|}{ Kimchi cabbage powder } \\
\hline & & Linoleic acid & a-Linolenic acid & Linoleic acid & a-Linolenic acid \\
\hline \multirow[t]{5}{*}{1} & 1 & $6447 \pm 136$ & $19947 \pm 820$ & $1387 \pm 30$ & $9441 \pm 338$ \\
\hline & 2 & $6350 \pm 187$ & $19694 \pm 793$ & $1362 \pm 30$ & $9523 \pm 376$ \\
\hline & 3 & $6385 \pm 131$ & $19882 \pm 753$ & $1366 \pm 30$ & $9444 \pm 306$ \\
\hline & Mean & 6394 & 19841 & 1372 & 9469 \\
\hline & $S D^{b}$ & $49(0.77 \%)$ & $131(1.32 \%)$ & $13(0.98 \%)$ & $47(0.49 \%)$ \\
\hline \multirow[t]{5}{*}{2} & 1 & $6248 \pm 173$ & $19657 \pm 368$ & $1373 \pm 32$ & $9447 \pm 281$ \\
\hline & 2 & $6338 \pm 173$ & $19850 \pm 482$ & $1365 \pm 29$ & $9673 \pm 144$ \\
\hline & 3 & $6429 \pm 177$ & $19337 \pm 592$ & $1337 \pm 33$ & $9319 \pm 180$ \\
\hline & Mean & 6338 & 19615 & 1358 & 9480 \\
\hline & $S D^{b}$ & $91(1.43 \%)$ & $259(1.32 \%)$ & $19(1.39 \%)$ & 179 (1.89\%) \\
\hline \multirow[t]{5}{*}{3} & 1 & $6301 \pm 246$ & $19980 \pm 470$ & $1320 \pm 56$ & $9645 \pm 224$ \\
\hline & 2 & $6333 \pm 244$ & $19764 \pm 461$ & $1318 \pm 51$ & $9609 \pm 227$ \\
\hline & 3 & $6350 \pm 252$ & $19965 \pm 493$ & $1349 \pm 57$ & $9698 \pm 226$ \\
\hline & Mean & 6328 & 19903 & 1329 & 9651 \\
\hline & $S D^{b}$ & $25(0.39 \%)$ & $121(0.61 \%)$ & $17(1.31 \%)$ & $45(0.46 \%)$ \\
\hline \multicolumn{2}{|c|}{ Overall mean } & 6353 & 19786 & 1353 & 9533 \\
\hline \multicolumn{2}{|c|}{ SD among periods ${ }^{c}$} & $36(0.56 \%)$ & $152(0.77 \%)$ & $22(1.61 \%)$ & $102(1.07 \%)$ \\
\hline
\end{tabular}

"The number following " \pm " is the expanded uncertainty with a $95 \%$ confidence level for the measurement value.

${ }^{\mathrm{b}}$ The standard deviation (SD) was determined among three values obtained within the period.

${ }^{\mathrm{C}}$ The standard deviation (SD) was determined among three mean values obtained at different time points.

implies the ID-GC/MS approach is commutable with the analytical method used by the collaborating laboratories.

The limit of detection (LOD) was evaluated by testing the standard solutions of the target essential fatty acids with signal-to-noise ratio $(\mathrm{S} / \mathrm{N})$ of 3 . The LODs of linoleic acid and $\alpha$-linolenic acid using the ID-GC/MS method were $0.15 \mathrm{mg} / \mathrm{kg}$ and $0.1 \mathrm{mg} / \mathrm{kg}$, respectively. The limit of quantification $(\mathrm{S} / \mathrm{N}=10)$ of linoleic acid and $\alpha$-linolenic acid were $0.5 \mathrm{mg} / \mathrm{kg}$ and $0.3 \mathrm{mg} / \mathrm{kg}$, respectively.

\section{Application of ID-GC/MS method to commercial products} The developed ID-GC/MS method was applied to analyze essential fatty acid contents in commercially available products including cereal and vegetable powders purchased from the local market. Of the various fatty acids, cereal products contained the highest amount of linoleic acid. As shown in Table 3, the commercial rice and wheat flours contain similar amounts of linoleic acid as those observed in the homemade homogenized rice and wheat samples. The rice snacks made with $100 \%$ rice show differences in linoleic acid contents depending on the rice varieties. For the vegetable products, linoleic acid and $\alpha$ - linolenic acid are found as the two major essential fatty acids. Similar to the measurement results for the homogenized vegetables, $\alpha$-linolenic acid contents are much higher than those of linoleic acid extracted from broccoli and green tea powders (Table 3).

Table 3 Linoleic acid and a-linolenic acid contents of commercial cereal and leaf vegetable products by the developed ID-GC/MS method

\begin{tabular}{llll}
\hline Samples & & \multicolumn{2}{l}{ Results by ID-GC/MS (mg/kg) ${ }^{\text {a }}$} \\
\cline { 2 - 3 } Rice flour & A & $3354 \pm 54$ & a-Linolenic acid \\
\hline & B & $3167 \pm 67$ & - \\
Wheat flour & C & $3772 \pm 68$ & - \\
& A & $6699 \pm 53$ & - \\
Rice snack & B & $6771 \pm 79$ & - \\
Croccoli powder & C & $7207 \pm 110$ & - \\
Green tea powder & A & $4196 \pm 36$ & - \\
\hline
\end{tabular}

"The number following " \pm " is the expanded uncertainty with a $95 \%$ confidence level for the measurement value. 
Among the vegetable samples, spinach contains the highest contents of both essential fatty acids.

\section{Conclusions}

An ID-GC/MS method, which can be used as a reference method for the accurate determination of essential fatty acid contents in cereals and green vegetables is described. Isotope-labeled fatty acids as internal standards were added ahead of the sample preparation steps for the GC analysis, which eliminate possible bias resulting from sample preparation and instrumental measurements. The developed ID-GC/MS method is validated by determining the repeatability, reproducibility, and measurement uncertainty. The SI-traceable measurement results of the essential fatty acids in raw food matrices provides a higher-order metrological quality. A comparison of the target fatty acid analysis results among the collaborating laboratories shows a good agreement within the expanded uncertainties. The developed method can be used to evaluate the reliability of analytical procedures for the quantitative analysis of fatty acids by comparing the measurement results. Additionally, cereal and vegetable CRMs are currently being developed using the ID-GC/MS approach.

\section{Abbreviations \\ ID-GC/MS: Isotope dilution-gas chromatography/mass spectrometry; GC: Gas chromatography; FAMEs: Fatty acid methyl esters; MS: Mass spectrometry; CCQM: Comité Consultatif pour la Quantité de Matière; IDMS: Isotope dilution mass spectrometry; SI: International System; CRMs: Certified Reference Materials; BHT: Butylated hydroxytoluene; FFAs: Free fatty acids; El: Electron impact ionization; SIM: Selected ion monitoring; TICs: Total ion chromatograms; RSD: Relative standard deviations; GC-FID: Gas chromatography-flame ionization detection; LOD: Limit of detection}

\section{Authors' contributions}

SL designed and carried out ID-GC/MS analysis and wrote the manuscript. DKL conducted sample preparation. SYB carried out purity analysis. DS, JSP, BMK, and JW carried out the comparative analysis. JL and BK supervised the entire work and edited the manuscript. All authors have read, corrected, and approved the final manuscript.

\section{Funding}

This study was supported by the Korea Research Institute of Standards and Science under the projects 'Nutritional Metrology Program' and 'Establishment of Measurement Standards for Organic Analysis', grants 1801107 and 20011034, respectively.

\section{Availability of data and materials}

Not applicable.

\section{Competing interests}

The authors declare that they have no competing interests.

\section{Author details}

'Division of Chemical and Biological Metrology, Korea Research Institute of Standards and Science (KRISS), Daejeon 34113, Republic of Korea. ${ }^{2}$ Food Analysis Center, Korea Food Research Institute, Wanju-Gun, Jeollabuk-do 55365, Republic of Korea. ${ }^{3}$ Food Safety Center, Namyang Dairy Product Company, Sejong 30055, Republic of Korea.
Received: 8 June 2020 Accepted: 11 August 2020

Published online: 25 August 2020

\section{References}

An S, Yim Y-H, Kim B. Development of gas chromatography/mass spectrometry for the determination of essential fatty acids in food supplemental oil products. Mass Spectrom Lett. 2013;4:75-8.

Arbuckle LD, MacKinnon MJ, Innis SM. Formula 18:2(n-6) and 18:3(n-3) content and ratio influence long-chain polyunsaturated fatty acids in the developing piglet liver and central nervous system. J Nutr. 1994;124:289-98.

Choi J, Hwang E, So HY, Kim B. An uncertainty evaluation for multiple measurements by gum. Accredit Qual Assur. 2003;8:13-5.

Connor WE. A-linolenic acid in health and disease. Am J Clin Nutr. 1999:69:827-8.

Cruz-Hernandez C, Goeuriot S, Giuffrida F, Thakkar SK, Destaillats F. Direct quantification of fatty acids in human milk by gas chromatography. J Chromatogr A. 2013;1284:174-9.

Folch J, Lees M, Sloane Stanley GH. A simple method for the isolation and purification of total lipides from animal tissues. J Biol Chem. 1957;226:497509.

Harwood JL. Fatty acid metabolism. Ann Rev Plant Physiol Plant Mol Biol. 1988; 39:101-38.

Holman RT. The slow discovery of the importance of $\omega 3$ essential fatty acids in human health. J Nutr. 1998;128:427S-33S.

Innis SM. Essential fatty acids in growth and development. Prog Lipid Res. 1991; 30:39-103.

Innis SM. Dietary omega 3 fatty acids and the developing brain. Brain Res. 2008; 1237:35-43.

Kim B-J, Hwang E-J, So H-Y, Son E-K, Kim Y-S. Development of a model system of uncertainty evaluations for multiple measurements by isotope dilution mass spectrometry: Determination of folic acid in infant formula. Bull Korean Chem Soc. 2010;31:3139-44.

Kim S-H, Lee J, Seonghee A, Song Y-S, Kim D-K, Kim B. Purity assessment of organic reference materials with a mass balance method: A case study of endosulfan-ii. Bull Korean Chem Soc. 2013;34:531-8.

Komaitis ME, Melissari-Panagiotou E. Lipid levels in cabbage leaves (brassica oleracea). J Sci Food Agric. 1990;50:571-3.

Lee J, Kim B. Mass balance method for purity assessment of organic reference materials: For thermolabile materials with Ic-uv method. Bull Korean Chem Soc. 2014;35:3275-9.

Lee J, Park K, Lee S, Choe E. Lipid changes of freeze-dried spinach by various kinds of oxidation. J Food Sci. 2000;65:1290-5.

Lee J, Song Y-S, H-j S, Kim B. Isotope dilution-liquid chromatography/mass spectrometric method for the determination of riboflavin content in multivitamin tablets and infant formula. J Food Compost Anal. 2016;50:4954.

Lee H, Lee J, Choi K, Kim B. Development of isotope dilution-liquid chromatography/tandem mass spectrometry for the accurate determination of trans- and cis-vitamin k1 isomers in infant formula. Food Chem. 2017;221: 729-36.

Lee S, Lee J, Ahn S, Baek S-Y, Kim B. Determination of fatty acid contents in infant formula by isotope dilution-gas chromatography/mass spectrometry. J Food Compost Anal. 2019a;80:33-9.

Lee J, Kim B, Lee SY, Choi J, Kang D, Lee H, Choi K, Lee H, Sim H-J, Baek S-Y, Lee H, Hyung S-W, Ahn S, Seo D, Hwang J, Park J-S, Kwak B-M, Won J. Development of an infant formula certified reference material for the analysis of organic nutrients. Food Chem. 2019b;298:125088.

Neuringer M, Connor WE, CVan P, Barstad L. Dietary omega-3 fatty acid deficiency and visual loss in infant rhesus monkeys. J Clin Invest. 1984;73: 272-6.

Quinn TJ. Primary methods of measurement and primary standards. Metrologia. 1997;34:61-5.

Richter W. Primary methods of measurement in chemical analysis. Accredit Qual Assur. 1997;2:354-9.

Sharpless K, Lindstrom RM, Nelson BC, Phinney KW, Rimmer CA, Sander LC, Schantz MM, Spatz RO, Thomas JB, Turk GC, Wise SA, Wood LJ, Yen JH Preparation and characterization of standard reference material 1849 infant/ adult nutritional formula. J AOAC Int. 2019;93:1262-74.

Shin H, Kim B, Lee J. Investigation of isotope dilution mass spectrometric (ID-MS) method to determine niacin in infant formula, breakfast cereals and multivitamins. Food Chem. 2013;138:1109-15. 
Takemoto Y, Suzuki Y, Horibe R, Shimozawa N, Wanders RJA, Kondo N. Gas chromatography/mass spectrometry analysis of very long chain fatty acids, docosahexaenoic acid, phytanic acid and plasmalogen for the screening of peroxisomal disorders. Brain Dev. 2003;25:481-7.

Taylor TC, Nelson JM. Fat associated with starch. J Am Chem Soc. 1920;42:1726-38.

Uauy R, Dangour AD. Nutrition in brain development and aging: Role of essential fatty acids. Nutr Rev. 2006;64:S24-33.

Wise SA, Phillips MM. Evolution of refer ence materials for the determination of organic nutrients in food and dietary supplements-a critical review. Anal Bioanal Chem. 2019;411:97-127.

Yasumatsu K, Moritaka S. Fatty acid compositions of rice lipid and their changes during storage. Agric Biol Chem. 1964;28:257-64.

Yoshida H, Tanigawa T, Yoshida N, Kuriyama I, Tomiyama Y, Mizushina Y. Lipid components, fatty acid distributions of triacylglycerols and phospholipids in rice brans. Food Chem. 2011;129:479-84.

\section{Publisher's Note}

Springer Nature remains neutral with regard to jurisdictional claims in published maps and institutional affiliations.

\section{Submit your manuscript to a SpringerOpen ${ }^{\circ}$ journal and benefit from:}

- Convenient online submission

- Rigorous peer review

- Open access: articles freely available online

High visibility within the field

- Retaining the copyright to your article

Submit your next manuscript at $\boldsymbol{\nabla}$ springeropen.com 